\title{
CONVOLUTIONS OF DISTRIBUTIONS WITH EXPONENTIAL AND SUBEXPONENTIAL TAILS
}

\author{
DAREN B. H. CLINE
}

(Received 10 August 1985; revised 24 September 1986)

Communicated by T. C. Brown

\begin{abstract}
Distribution tails $\bar{F}(t)=F(t, \infty)$ are considered for which $\bar{F}(t-u) \sim e^{a u} \bar{F}(t)$ and $\overline{F * F(t)}-2 d \bar{F}(t)$ as $t \rightarrow \infty$. A real analytic proof is obtained of a theorem by Chover, Wainger and Ney, namely that

$$
d=\int e^{\alpha u} F(d u) .
$$

In doing so, a technique is introduced which provides many other results with a minimum of analysis. One such result strengthens and generalizes the various known results on distribution tails of random sums.

Additionally, the closure and factorization properties for subexponential distributions are investigated further and extended to distributions with exponential tails.
\end{abstract}

1980 Mathematics subject classification (Amer. Math. Soc.): $60 \mathrm{E} 05$.

Keywords: convolution tails, exponential tails, subexponential tails.

\section{Introduction}

Throughout this work we will use distribution tails and denote them, for example, $\bar{F}(t)=F(t, \infty)$, where $F$ is a finite nonnegative measure. For convenience, the distributions will have all their support on $[0, \infty)$. In application, these may be probability distributions (that is, total measure equal to 1) but we will not always assume so. Our convention is that integrals will exclude (include) the lower (upper) endpoint. The exception to this rule is when the lower endpoint is zero or for $\bar{F}(0)=F[0, \infty)$. If a function $A(t)$ is regularly varying with exponent $\rho$, we

C. 1987 Australian Mathematical Society $0263-6115 / 87 \$ A 2.00+0.00$ 
write $A \in R V_{\rho}$. Also, we will signify $A_{1} \sim k A_{2}$ and $A_{1} \leq k A_{2}\left(A_{1}=o\left(A_{2}\right)\right.$ for $k=0$ ) when

$$
\lim _{t \rightarrow \infty} \frac{A_{1}(t)}{A_{2}(t)}=k \text { and } \quad \limsup _{t \rightarrow \infty} \frac{A_{1}(t)}{A_{2}(t)} \leqslant k, \text { respectively. }
$$

Of course, $k A_{2} \geq A_{1}$ is the same as $A_{1} \leq k A_{2}$.

The purpose of this paper is to consider these relations ( $\sim$ and $\leq$ ), applied to distribution tails and to their Stieltjes convolution $\overline{F * G}(t)=$ $\iint_{x+y>t} F(d x) G(d y)$. Multiple convolutions of $F$ with itself will be denoted $F^{* n}$ and $F^{* 0}$ is the distribution with unit mass at zero. The discussion is limited to the following classes of distributions.

The class $L_{\alpha}, \alpha>0$, consists of all distributions $F$ such that $\bar{F}(t-u) \sim e^{\alpha u} \bar{F}(t)$ for each $u$. One may easily see that $F \in L_{\alpha}$ if and only if $\bar{F}(\ln t) \in R V_{-\alpha}$. When $\alpha>0, F$ is said to have exponential tail.

$F$ is called convolution equivalent $\left(F \in S_{\alpha}\right)$ if $F \in L_{\alpha}$ and $\overline{F * F}-2 d \bar{F}$ for some finite positive $d$. If $F \in S_{0}(\alpha=0)$, it is called subexponential. For example, $\bar{F} \in R V_{-\rho}$ implies $F \in S_{0}$.

The classes $S_{0}$ and $S_{\alpha}$ have received attention with applications to branching processes (Chistykov (1964), Chover, Wainger and Ney (1973a, b)), to renewal theory (Embrechts and Goldie (1982)), to infinite variance time series (Davis and Resnick (1985b) and to stable attraction of sums of products (Cline (1986)). References to further applications in queueing theory, random walks and infinite divisibility may be found in Embrechts and Goldie (1982). Conditions sufficient for $F \in S_{\alpha}$ are given in Cline (1986).

Chover, Wainger and Ney (1973a) first defined the class $S_{\alpha}$ in terms of the probability masses on the lattice of nonnegative integers:

$$
f_{n}=F\{n\} \quad \text { and } f_{n} * f_{n}=F * F\{n\}, \quad n=0,1,2, \ldots
$$

That is, $F \in S_{\alpha}$ if $f_{n} \sim e^{\alpha} f_{n+1}$ and $f_{n} * f_{n} \sim 2 d f_{n}$. They gave another, similar, definition for densities of an absolutely continuous $F$. In their first paper, they use a Banach algebra approach to demonstrate

$$
\text { If } F \in S_{\alpha} \text { then } d=m \text {, where } m=\int_{0}^{\infty} e^{\alpha u} F(d u) \text {. }
$$

Chover, Winger and Ney (1973a, b) also verify (1.1) for the "global" version, where $S_{\alpha}$ is as we have defined it, in terms of distribution tails. Rudin (1973) and Embrechts (1983) have given a real analytic proof for the lattice version of (1.1). In this paper, Theorem 2.9 provides an elementary real analytic proof for the distribution tails version. Embrechts' lattice version is a special case. However, the version for densities is not (see the Conclusion). 
In the course of pursuing this objective we devise a technique to calculate asymptotic relationships between convolution tails, which enables us to obtain several other known or partially known results with ease. In particular, Theorem 2.13 states in its most complete form a result for the measure $H=\sum_{0}^{\infty} \lambda_{n} F^{* n}$, namely that with appropriate conditions on $\lambda_{n}$, the following are equivalent.

(i) $F \in S_{\alpha}$.

(ii) $\bar{H} \sim c \bar{F}$ where $c=\sum_{0}^{\infty} n \lambda_{n} m^{n-1}$.

(iii) $H \in S_{\alpha}$ and $\bar{F} \neq o(\bar{H})$.

That (i) implies (ii) is the main objective in Chover, Wainger and Ney (1973a, b). Embrechts, Goldie and Veraverbeke (1979) and Embrechts and Goldie (1982) prove the equivalency for special cases of $\lambda_{n}$. Rudin (1973) and Embrechts (1983) also prove (i) implies (ii) for lattice $F$.

Embrechts and Goldie (1980) consider the question, if $F_{1} \in S_{0}$ and $F_{2} \in S_{0}$ then is $F_{1} * F_{2} \in S_{0}$ ? The question remains unanswered, but they show that $F_{1} * F_{2} \in S_{0}$ is equivalent to $\overline{F_{1} * F_{2}}-m_{2} \bar{F}_{1}+m_{1} \bar{F}_{2}$, where $m_{j}=\bar{F}_{j}(0)$, and that each is equivalent to $a_{1} F_{1}+a_{2} F_{2} \in S_{0}$, where $a_{1}>0, a_{2}>0$. In Theorem 3.4, we extend this result to the class $S_{\alpha}$ (with $m_{j}=\int_{0}^{\infty} e^{\alpha u} F_{j}(d u)$ ) and strengthen it in two important respects. First, if $F_{1}, F_{2} \in L_{\alpha}$ then either $F_{1} \in S_{\alpha}$ or $F_{2} \in S_{\alpha}$ is a consequence of $F_{1} * F_{2} \in S_{\alpha}$. Second, if $F_{1} \in S_{\alpha}$ and either $F_{2} \in S_{\alpha}$ or $\bar{F}_{2}=o\left(\bar{F}_{1}\right)$, the statement $\overline{F_{1} * F_{2}} \sim m_{2} \bar{F}_{1}+m_{1} \bar{F}_{2}$ may be weakened to $\bar{F}_{1} * F_{2} \sim a_{1} \bar{F}_{1}+a_{2} \bar{F}_{2}$ for some $a_{1}, a_{2}$, and it will still retain its power to imply $F_{1} * F_{2} \in S_{\alpha}$.

Finally, we will provide actual conditions for which $\overline{F_{1} * F_{2}} \sim m_{2} \bar{F}_{1}+m_{1} \bar{F}_{2}$. The well-known result of Feller (1971, page 278) states that this holds for distributions with regularly varying tails. Embrechts and Goldie (1980) show that if $F_{1} \in S_{0}$, then $\sup _{t} \bar{F}_{1}(t) / \bar{F}_{2}(t)<\infty$ suffices. In fact, for $F_{j} \in S_{\alpha}$, it is sufficient to have $\bar{F}_{1} / \bar{F}_{2} \in R V_{\rho}$ for some $\rho$. This and a more general condition appear in Theorem 3.5.

\section{Convolution equivalency}

We state at the outset that the reader should keep Theorem 2.9 in mind when considering results 2.5 through 2.8 in this section. Once Theorem 2.9 has been proved we will restate these in a more precise form. Many of these have been proven elsewhere, but only after Theorem 2.9 was verified. Our approach is to work in the other direction.

We start with several lemmas.

LEMMA 2.1. Let $A_{j}(t)$ be positive functions.

(i) If $A_{3} \leq A_{1} \leq c A_{2}$, then $A_{1}+A_{2} \leq A_{3}+A_{4}$ implies $A_{2} \leq A_{4}$.

(ii) If $A_{3} \sim A_{1} \leq c A_{2}$, then $A_{1}+A_{2} \sim A_{3}+A_{4}$ implies $A_{2} \sim A_{4}$. 
(iii) $a_{1} A_{1}+a_{2} A_{2} \sim b_{1} A_{1}+b_{2} A_{2}$ implies $a_{1}=b_{1}$ or

$$
A_{1} \sim \frac{b_{2}-a_{2}}{a_{1}-b_{1}} A_{2}
$$

(iv) If $A_{1} \lesssim A_{3}, A_{2} \lesssim A_{4}$, then $A_{1}+A_{2} \sim A_{3}+A_{4}$ implies one of the following must hold.

$$
\begin{array}{ll}
A_{1} \sim A_{3}, & A_{2} \sim A_{4}, \\
A_{1} \sim A_{3}, & A_{4}=o\left(A_{1}\right), \text { or } \\
A_{2} \sim A_{4}, & A_{3}=o\left(A_{2}\right) .
\end{array}
$$

Proof. Straightforward.

LeMMA 2.2. Suppose $F_{1}, F_{2} \in L_{\alpha}$.

(i) (Embrechts and Goldie, 1982) The transforms $m_{j}(\gamma)=\int_{0}^{\infty} e^{\gamma u} F_{j}(d u)$ have their singularity at $\gamma=\alpha$.

(ii) If $m_{j}=m_{j}(\alpha)$, then $\overline{F_{1} * F_{2}} \geq m_{2} \bar{F}_{1}+m_{1} \bar{F}_{2}$.

In particular, if $\overline{F_{1} * F_{1}}-2 d_{1} \bar{F}_{1}$ with $d_{1}<\infty$, then $m_{1} \leqslant d_{1}$.

Proof. (ii) Although this has been observed by several authors, we repeat it because it is such a basic result. Write

$$
\overline{F_{1} * F_{2}}(t)=\int_{0}^{t / 2} \bar{F}_{1}(t-u) F_{2}(d u)+\int_{0}^{t / 2} \bar{F}_{2}(t-u) F_{1}(d u)+\bar{F}_{1}(t / 2) \bar{F}_{2}(t / 2) .
$$

Fatou's lemma gives

$$
\liminf _{t \rightarrow \infty} \int_{0}^{t / 2} \frac{\bar{F}_{1}(t-u)}{\bar{F}_{1}(t)} F_{2}(d u) \geqslant \int_{0}^{\infty} e^{\alpha u} F_{2}(d u)=m_{2}
$$

The second term is handled similarly and the third is ignored.

Lemma 2.2(ii) holds for all distributions when $m_{j}$ is replaced with $\vec{F}_{j}(0)=$ $F_{j}[0, \infty)$. In fact, it may be shown that if $\overline{F_{1} * F_{2}} \sim \bar{F}_{2}(0) \bar{F}_{1}+\bar{F}_{1}(0) \bar{F}_{2}$ and $\bar{F}_{2} \leqslant c \bar{F}_{1}$ then $F_{1} \in L_{0}$. (See Chistykov (1964) for a proof when $F_{1}=F_{2}$.)

Throughout the paper, we will use $m_{j}=\int_{0}^{\infty} e^{\alpha u} F_{j}(d u)$ whenever $F_{j} \in L_{\alpha}$ and many of our theorems will assume this parameter to be finite. When referring to distributions $F, G$ or $H$, the parameter will be denoted $m\left(=m_{F}\right), m_{G}$ or $m_{H}$.

The next lemma shows that $L_{\alpha}$ is closed under convolutions.

LEMMA 2.3. (i) (Embrechts and Goldie, 1980). If $F_{j} \in L_{\alpha}$, then $F_{1} * F_{2} \in L_{\alpha}$.

(ii) Assume $\lambda_{n} \geqslant 0$ and $\sum_{0}^{\infty} \lambda_{n}(\vec{F}(0))^{n}<\infty$. If $F \in L_{\alpha}$ and $H=\sum_{0}^{\infty} \lambda_{n} F^{* n}$, then for $u>0, \bar{H}(t-u) \leq e^{\alpha u} \bar{H}(t)$. In particular, if $\alpha=0$ then $H \in L_{0}$. 
Proof. (i) We have simplified slightly the proof by Embrechts and Goldie. Fix $u>0$. For large enough $s$ and $t_{0} \geqslant 2 s$,

and

$$
(1-\varepsilon) e^{\alpha u} \bar{F}_{1}(t) \leqslant \bar{F}_{1}(t-u) \leqslant(1+\varepsilon) e^{\alpha u} \bar{F}_{1}(t), \quad t \geqslant s,
$$

$$
(1-\varepsilon) e^{\alpha v} \bar{F}_{2}(t) \leqslant \bar{F}_{2}(t-v) \leqslant(1+\varepsilon) e^{\alpha v} \bar{F}_{2}(t),
$$$$
t \geqslant t_{0} \text { and } v=u, s \text { or } u+s .
$$

Embrechts and Goldie show that for all $t \geqslant t_{0}$,

$$
\overline{F_{1} * F_{2}}(t-u) \leqslant(1+\varepsilon) e^{\alpha u} \overline{F_{1} * F_{2}}(t) .
$$

Thus,

$$
\limsup _{t \rightarrow \infty} \frac{\overline{F_{1} * F_{2}}(t-u)}{\overline{F_{1} * F_{2}}(t)} \leqslant e^{\alpha u}
$$

Similarly, for $t \geqslant t_{0}$,

$$
\begin{aligned}
\overline{F_{1} * F_{2}}(t-u) & \geqslant(1-\varepsilon) e^{\alpha u} \overline{F_{1} * F_{2}}(t)-\int_{t-u-s}^{t-s} \bar{F}_{1}(t-u-v) F_{2}(d v) \\
& \geqslant(1-\varepsilon) e^{\alpha u} \overline{F_{1} * F_{2}}(t)-\bar{F}_{1}(s-u) \bar{F}_{2}(t-u-s) \\
& \geqslant(1-\varepsilon) e^{\alpha u} \overline{F_{1} * F_{2}}(t)-(1+\varepsilon)^{2} e^{2 \alpha u} e^{\alpha s} \bar{F}_{1}(s) \bar{F}_{2}(t) .
\end{aligned}
$$

Now, $\bar{F}_{2} \leq 1 / m_{1} \overline{F_{1} * F_{2}}$ by Lemma 2.2 (ii). Thus, if $m_{1}=\infty, \bar{F}_{2}=o\left(\overline{F_{1} * F_{2}}\right)$ and if $m_{1}<\infty$, then $e^{\alpha s} \bar{F}_{1}(s) \rightarrow 0$ as $s \rightarrow \infty$. In either case,

$$
\liminf _{t \rightarrow \infty} \frac{\overline{F_{1} * F_{2}}(t-u)}{\overline{F_{1} * F_{2}}(t)} \geqslant e^{\alpha u},
$$

which proves (i).

(ii) Apply (2.1) recursively, with $F_{1}=F, F_{2}=F^{* n-1}$. Thus

$$
\bar{F}^{* n}(t-u) \leqslant(1+\varepsilon) e^{\alpha u} \bar{F}^{* n}(t), \text { all } n \text {, all } t \geqslant t_{0} .
$$

Hence $\bar{H}(t-u) \leqslant(1+\varepsilon) e^{\alpha u} \bar{H}(t)$ and

$$
\limsup _{t \rightarrow \infty} \frac{\bar{H}(t-u)}{\bar{H}(t)} \leqslant e^{\alpha u} .
$$

In case $\alpha=0$, then $H \in L_{0}$ follows because $\bar{H}$ is nonincreasing.

Although we have not given sufficient conditions here for $H$ to be in $L_{\alpha}$ (except when $\alpha=0$ ), we will later show that under certain assumptions $F \in S_{\alpha}$ implies $H \in S_{\alpha}$. The next lemma, a new result, is the basis for all our later results. 
LEMMA 2.4. Suppose $F_{j} \in L_{\alpha}$ and $m_{j}<\infty, j=1,2,3,4$.

(i) If $\bar{F}_{1} \leqslant \bar{F}_{3}$ and $\bar{F}_{2} \leqslant \bar{F}_{4}$, then $\overline{F_{1} * F_{2}}+m_{4} \bar{F}_{3}+m_{3} \bar{F}_{4} \leqslant \overline{F_{3} * F_{4}}+$ $m_{2} \bar{F}_{1}+m_{1} \bar{F}_{2}$.

(ii) If $\bar{F}_{1} \sim \bar{F}_{3}$ and $\bar{F}_{2} \sim \bar{F}_{4}$, then $\overline{F_{1} * F_{2}} \sim \overline{F_{3} * F_{4}}+\left(m_{2}-m_{4}\right) \bar{F}_{3}+$ $\left(m_{1}-m_{3}\right) \bar{F}_{4}$.

Proof. We will prove only (i). The proof for (ii) uses a double application of (i) and an appeal to Lemma 2.1(ii).

We may write

$$
\begin{aligned}
\overline{F_{1} * \bar{F}_{2}}(t)= & \int_{0}^{s} \bar{F}_{1}(t-u) F_{2}(d u)+\int_{0}^{s} \bar{F}_{2}(t-u) F_{1}(d u) \\
& +\int_{s}^{t-s} \bar{F}_{1}(t-u) F_{2}(d u)+\bar{F}_{1}(s) \bar{F}_{2}(t-s) . \\
\overline{F_{3} * F_{4}}(t)= & \int_{0}^{s} \bar{F}_{3}(t-u) F_{4}(d u)+\int_{0}^{s} \bar{F}_{4}(t-u) F_{3}(d u) \\
& +\int_{s}^{t-s} \bar{F}_{4}(t-u) F_{3}(d u)+\bar{F}_{4}(s) \bar{F}_{3}(t-s) .
\end{aligned}
$$

For large enough $s, \bar{F}_{1}(u) \leqslant(1+\varepsilon) \bar{F}_{3}(u)$ and $\bar{F}_{2}(u) \leqslant(1+\varepsilon) \bar{F}_{4}(u)$, all $u \geqslant s$. Thus for $t>2 s$,

$$
\begin{aligned}
\int_{s}^{t-s} \bar{F}_{1}(t-u) & F_{2}(d u)+\bar{F}_{1}(s) \bar{F}_{2}(t-s) \\
& \leqslant(1+\varepsilon)\left[\int_{s}^{t-s} \bar{F}_{3}(t-u) F_{2}(d u)+\bar{F}_{3}(s) \bar{F}_{2}(t-s)\right] \\
& =(1+\varepsilon)\left[\int_{s}^{t-s} \bar{F}_{2}(t-u) F_{3}(d u)+\bar{F}_{2}(s) \bar{F}_{3}(t-s)\right] \\
& \leqslant(1+\varepsilon)^{2}\left[\int_{s}^{t-s} \bar{F}_{4}(t-u) F_{3}(d u)+\bar{F}_{4}(s) \bar{F}_{3}(t-s)\right]
\end{aligned}
$$

Again for large $s$,

$$
\left|\int_{0}^{s} e^{\alpha u} F_{j}(d u)-m_{j}\right|<\varepsilon \min _{i} m_{i}, \quad j=1,2,3,4 .
$$

Fixing $s$, we may choose $t$ large enough so that

$$
\left|\int_{0}^{s} \frac{\bar{F}_{i}(t-u)}{\bar{F}_{i}(t)} F_{j}(d u)-\int_{0}^{s} e^{\alpha u} F_{j}(d u)\right|<\varepsilon m_{j}, \quad i, j=1,2,3,4, i \neq j .
$$

From (2.2), (2.3) and (2.4) we thus have

$$
\begin{aligned}
\overline{F_{1} * F_{2}}(t)-(1+ & 2 \varepsilon)\left(m_{2} \bar{F}_{1}(t)+m_{1} \bar{F}_{2}(t)\right) \\
& \leqslant(1+\varepsilon)^{2}\left[\overline{F_{3} * F_{4}}(t)-(1-2 \varepsilon)\left(m_{4} \bar{F}_{3}(t)+m_{3} \bar{F}_{4}(t)\right)\right] .
\end{aligned}
$$


This implies

$$
\begin{aligned}
& \left(1-4 \varepsilon^{2}\right)\left[\overline{F_{1} * F_{2}}(t)+m_{4} \bar{F}_{3}(t)+m_{3} \bar{F}_{4}(t)\right] \\
& \quad \leqslant(1+\varepsilon)^{2}\left[\overline{F_{3} * F_{4}}(t)+m_{2} \bar{F}_{1}(t)+m_{1} \bar{F}_{2}(t)\right] .
\end{aligned}
$$

Since $\varepsilon$ is arbitrary, then

$$
\overline{F_{1} * F_{2}}+m_{4} \bar{F}_{3}+m_{3} \bar{F}_{4} \leq \overline{F_{3} * F_{2}}+m_{2} \bar{F}_{1}+m_{1} \bar{F}_{2} .
$$

The power of Lemma 2.4 can be seen in the next three corollaries.

Corollary 2.5. If $F_{1} \in L_{\alpha}, m_{1}<\infty$ and $\bar{F}_{1} \sim \bar{F}_{2}$, then

$$
\begin{aligned}
& \overline{F_{1} * F_{2}} \sim \overline{F_{1} * F_{1}}+\left(m_{2}-m_{1}\right) \bar{F}_{1} \quad \text { and } \\
& \overline{F_{2} * F_{2}} \sim \overline{F_{1} * F_{1}}+2\left(m_{2}-m_{1}\right) \bar{F}_{1} .
\end{aligned}
$$

In particular, if $\overline{F_{1} * F_{1}}-2 d_{1} \bar{F}_{1}$, then $\overline{F_{2} * F_{2}} \sim 2\left(m_{2}+d_{1}-m_{1}\right) \bar{F}_{2}$.

Proof. The first two relationships are straightforward applications of Lemma 2.4(ii). The third follows immediately from the second.

We see, therefore, that $S_{\alpha}$ is closed under asymptotic tail equivalency.

Corollary 2.6. Let $F \in L_{\alpha}$ and $m=\int_{0}^{\infty} e^{\alpha u} F(d u)<\infty$.

(i) Then $\bar{F}^{* n} \geq m^{n-2} \overline{F * F}+(n-2) m^{n-1} \bar{F} \geq n m^{n-1} \bar{F}$.

(ii) If $\overline{F * F} \leq 2 d \bar{F}(\overline{F * F} \geq 2 d \bar{F})$, then $\bar{F}^{* n} \leq a_{n} \bar{F}\left(\bar{F}^{* n} \geq a_{n} \bar{F}\right)$, where

$$
a_{n}= \begin{cases}n m^{n-1}, & \text { if } d=m, \\ \frac{(2 d-m)^{n}-m^{n}}{2(d-m)}, & \text { if } d \neq m .\end{cases}
$$

Proof. (i) By repeated application of Lemma 2.2(ii),

$$
\begin{aligned}
\bar{F}^{* n} & \geq m \bar{F}^{* n-1}+m^{n-1} \bar{F} \\
& \geq m^{2} \bar{F}^{* n-2}+2 m^{n-1} \bar{F} \\
\cdots & \geq m^{n-2} \overline{F * F}+(n-2) m^{n-1} \bar{F} \\
& \geq n m^{n-1} \bar{F} .
\end{aligned}
$$

(ii) Assume $\overline{F * F} \leq 2 d \bar{F}$. The proof is by induction and is similar when $\overline{F * F} \geq 2 d \bar{F}$. Suppose $\bar{F}^{* n} \leq a_{n} \bar{F}$. Then using Lemma 2.4(i) and Lemma 2.1(ii),

$$
\begin{aligned}
\bar{F}^{* n+1} & =\overline{F^{* n} * F} \leqslant a_{n} \overline{F * F}+\left(m^{n}-a_{n} m\right) \bar{F} \\
& \leq\left((2 d-m) a_{n}+m^{n}\right) \bar{F}=a_{n+1} \bar{F} .
\end{aligned}
$$


Once we establish that $d=m$, we will also be able to show that $\bar{F}^{* n} \sim n m^{n-1} \bar{F}$ is equivalent to $F \in S_{\alpha^{*}}$ (See Corollary 2.11.)

CoRollary 2.7. Suppose $F_{1} \in L_{\alpha}, \overline{F_{1} * F_{1}} \sim 2 d_{1} \bar{F}_{1}$ and $\lim _{t \rightarrow \infty} \bar{F}_{2}(t) / \bar{F}_{1}(t)=$ $k<\infty$. Then $\bar{G}=\overline{F_{1} * F_{2}} \sim\left(m_{2}+k\left(2 d_{1}-m_{1}\right)\right) \bar{F}_{1}$ and $G \in S_{\alpha}$.

Proof. The proof is valid whether $k=0$ or $k>0$. Since $\overline{F_{1}+F_{2}} \sim(1+k) \bar{F}_{1}$, then by Lemma 2.4 (ii),

$$
\begin{aligned}
\overline{F_{1} *\left(F_{1}+F_{2}\right)} & \sim(1+k) \overline{F_{1} * F_{1}}+\left(m_{2}-k m_{1}\right) \bar{F}_{1} \\
& \sim\left(2 d_{1}(1+k)+\left(m_{2}-k m_{1}\right)\right) \bar{F}_{1} .
\end{aligned}
$$

However, we also have $\overline{F_{1} *\left(F_{1}+F_{2}\right)}=\overline{F_{1} * F_{1}}+\bar{G} \sim 2 d_{1} \bar{F}_{1}+\bar{G}$. Applying Lemma 2.1(ii), $\bar{G} \sim\left(m_{2}+k\left(2 d_{1}-m_{1}\right)\right) \bar{F}_{1}=c \bar{F}_{1}$. By Corollary 2.5, $\overline{G * G} \sim$ $2\left(m_{G}+c\left(d_{1}-m_{1}\right)\right) \bar{G}$. Therefore $G \in S_{\alpha}$.

The proof of the next lemma follows that of a similar lemma in Chover, Wainger and Ney (1973b). (See Lemma 2.12.)

Lemma 2.8. Suppose $F \in L_{\alpha}, \overline{F * F} \sim 2 d \bar{F}, d<\infty$, and $m=\int_{0}^{\infty} e^{\alpha u} F(d u)$. Then for each $\varepsilon>0$ and some $K_{\varepsilon}$,

$$
\bar{F}^{* n}(t) \leqslant K_{\varepsilon}(2 d-m+\varepsilon)^{n-1} \bar{F}(t), \text { all } n \text {, all } t .
$$

Proof. For large enough $s$ and $t$,

$$
\int_{0}^{s} \frac{\bar{F}(t-u)}{\bar{F}(t)} F(d u) \geqslant m-\varepsilon / 4 .
$$

Fixing $s$, we may choose $t_{0}>s$ so that for $t \geqslant t_{0}$,

$$
\begin{aligned}
\frac{\overline{F * F}(t)}{\bar{F}(t)} & =\int_{0}^{t-s} \frac{\bar{F}(t-u)}{\bar{F}(t)} F(d u)+\int_{0}^{s} \frac{\bar{F}(t-u)}{\bar{F}(t)} F(d u)+\frac{\bar{F}(s) \bar{F}(t-s)}{\bar{F}(t)} \\
& \leqslant 2 d+\varepsilon / 4 .
\end{aligned}
$$

Thus $\int_{0}^{t-s} \bar{F}(t-u) F(d u)+\bar{F}(s) \bar{F}(t-s) \leqslant(2 d-m+\varepsilon / 2) \bar{F}(t), \quad t \geqslant t_{0}$. Choose

$$
K_{\varepsilon}=\max \left[\frac{2}{\varepsilon} \sup _{t \geqslant t_{0}} \frac{\bar{F}(t-s)}{\bar{F}(t)}, \frac{\bar{F}(0)}{\bar{F}\left(t_{0}\right)}\right]>1 .
$$

The conclusion obviously holds for $n=1$. We continue by induction. For $t \leqslant t_{0}$,

$$
\bar{F}^{* n+1}(t) \leqslant \bar{F}^{* n+1}(0) \leqslant m^{n} \bar{F}(0) \frac{\bar{F}(t)}{F\left(t_{0}\right)} \leqslant K_{\varepsilon}(2 d-m+\varepsilon)^{n} \bar{F}(t) .
$$




$$
\begin{aligned}
& \text { For } t>t_{0}, \\
& \begin{aligned}
\bar{F}^{* n+1}(t) \\
\quad=\int_{0}^{t-s} \bar{F}^{* n}(t-u) F(d u)+\bar{F}^{* n}(s) \bar{F}(t-s)+\int_{0}^{s} \bar{F}(t-u) F^{* n}(d u) \\
\quad \leqslant K_{\varepsilon}(2 d-m+\varepsilon)^{n-1}\left[\int_{0}^{t-s} \bar{F}(t-u) F(d u)+\bar{F}(s) \bar{F}(t-s)\right]+m^{n} \bar{F}(t-s) \\
\quad \leqslant\left[K_{\varepsilon}(2 d-m+\varepsilon)^{n-1}(2 d-m+\varepsilon / 2)+K_{\varepsilon}(\varepsilon / 2) m^{n}\right] \bar{F}(t) \\
\quad \leqslant K_{\varepsilon}(2 d-m+\varepsilon)^{n} \bar{F}(t) .
\end{aligned}
\end{aligned}
$$

We come now to our first major result, a new proof of a theorem by Chover, Wainger and Ney (1973a).

Theorem 2.9. If $F \in S_{\alpha}$, then $\overline{F * F} \sim 2 m \bar{F}$ where $m=\int_{0}^{\infty} e^{\alpha u} F(d u)$.

Proof. Suppose $\alpha>0$ and define

$$
\bar{F}_{0}(t)=\int_{t}^{\infty} e^{\alpha u} F(d u)
$$

It is easy to show that $F \in S_{\alpha}$ implies $F_{0} \in S_{0}$ (see Embrechts and Goldie (1982); they also show that the converse is not true), that $m_{0}(0)=m_{F}(\alpha)=m$ and that $\bar{F}_{0} * \bar{F}_{0}-2 d \bar{F}_{0}$. From these we see that it suffices to prove the theorem for the case $\alpha=0$.

From Lemma 2.2, we know that $d \geqslant m$. For $\lambda<1 / m$, define

$$
H_{\lambda}=\sum_{0}^{\infty} \lambda^{n} F^{* n}, \quad m_{\lambda}=\int_{0}^{\infty} H_{\lambda}(d u)=\frac{1}{1-m \lambda} .
$$

By Lemma 2.3(ii), $H_{\lambda} \in L_{0}$. If $\lambda<1 /(2 d-m)$, then Corollary 2.6(ii) and Lemma 2.8 combine with dominated convergence to give

$$
\bar{H}_{\lambda} \sim\left[\sum_{0}^{\infty} \lambda^{n}\left(\frac{(2 d-m)^{n}-m^{n}}{2(d-m)}\right)\right] \bar{F}=\frac{\lambda}{(1-m \lambda)(1-(2 d-m) \lambda)} \bar{F}
$$

(This is valid even when $d=m$.) On the other hand, by Fatou's Lemma, $\lambda \geqslant 1 /(2 d-m)$ implies

$$
\lim _{t \rightarrow \infty} \inf \frac{\bar{H}_{\lambda}(t)}{\bar{F}(t)} \geqslant \sum_{0}^{\infty} \lambda^{n}\left(\frac{(2 d-m)^{n}-m^{n}}{2(d-m)}\right)=\infty .
$$

Let

$$
\lambda_{0}=\sup \left\{\lambda: \bar{H}_{\lambda} \sim k_{\lambda} \bar{F} \text { for some } k_{\lambda}<\infty\right\}
$$


Since $k_{\lambda}$ must increase with $\lambda$, it is clear from (2.5) and (2.6) that $\lambda_{0}=$ $1 /(2 d-m)$ and that for all $\lambda<\lambda_{0}, \bar{H}_{\lambda} \sim k_{\lambda} \bar{F}$ for some finite $k_{\lambda}$.

Let

$$
\lambda_{1}=\sup \left\{\lambda: \overline{H_{\lambda} * H_{\lambda}} \sim 2 d_{\lambda} \bar{H}_{\lambda} \text { for some } d_{\lambda}<\infty\right\} .
$$

By Corollary 2.5, $d_{\lambda}=m_{\lambda}+k_{\lambda}(d-m)$ for all $\lambda<\lambda_{0}$. Thus $\lambda_{1} \geqslant \lambda_{0}$. Suppose $\lambda_{1}>\lambda_{0}$. (This requires $d>m$.) Let $\mu<\lambda_{0} \leqslant \lambda<\lambda_{1}$. Then $\bar{H}_{\mu} \sim k_{\mu} \bar{F}=o\left(\bar{H}_{\lambda}\right)$. Thus,

$$
\begin{aligned}
\overline{H_{\mu} * H_{\lambda}} & =\sum_{0}^{\infty} \sum_{0}^{\infty} \lambda^{j} \mu^{n-j} \bar{F}^{* n}=\sum_{0}^{\infty} \frac{\lambda^{n+1}-\mu^{n+1}}{\lambda-\mu} \bar{F}^{* n} \\
& =\left(\frac{\lambda}{\lambda-\mu}\right) \bar{H}_{\lambda}-\left(\frac{\mu}{\lambda-\mu}\right) \bar{H}_{\mu}-\left(\frac{\lambda}{\lambda-\mu}\right) \bar{H}_{\lambda} .
\end{aligned}
$$

But by Corollary 2.7, $\overline{H_{\mu} * H_{\lambda}} \sim m_{\mu} \bar{H}_{\lambda}$, a contradiction since $m_{\mu}<\lambda /(\lambda-\mu)$. Hence $\lambda_{1}=\lambda_{0}$.

Continue to assume $d>m$ and let $\lambda_{0}<\nu<\mu<\lambda<1 / m$. Since $\bar{H}_{\mu}=$ $o\left(\overline{H_{\mu} * H_{\mu}}\right)<\overline{H_{\mu} * H_{\lambda}}<(\lambda /(\lambda-\mu)) \bar{H}_{\lambda}$, it is clear that $\bar{H}_{\mu}=o\left(\bar{H}_{\lambda}\right)$ and $\overline{H_{\mu} * H_{\lambda}}$ $\sim(\lambda /(\lambda-\mu)) \overline{H_{\lambda}}$. Similarly, $\overline{H_{\nu} * H_{\lambda}} \sim(\lambda /(\lambda-\nu)) \bar{H}_{\lambda}$ and $\overline{H_{\nu} * H_{\mu}} \sim$ $(\mu /(\mu-\nu)) \bar{H}_{\mu}$. Applying Lemma $2.4($ ii) $)$

$$
\begin{aligned}
&\left(\frac{\mu}{\mu-\nu}\right)\left(\frac{\lambda}{\mu-\nu}\right) \bar{H}_{\lambda} \sim\left(\frac{\mu}{\mu-\nu}\right) \overline{H_{\mu} * H_{\lambda}} \\
& \quad=\overline{H_{\nu} * H_{\mu} * H_{\lambda}}+\left(\frac{\nu}{\mu-\nu}\right) \overline{H_{\nu} * H_{\lambda}} \\
& \sim\left(\frac{\mu}{\mu-\nu}\right) \overline{H_{\mu} * H_{\lambda}}+\left(m_{\mu} m_{\nu}-\frac{\mu}{\mu-\nu} m_{\mu}\right) \bar{H}_{\lambda}+\left(\frac{\nu}{\mu-\nu}\right) \overline{H_{\nu} * H_{\lambda}} \\
& \sim\left[\left(\frac{\mu}{\mu-\nu}\right)\left(\frac{\lambda}{\mu-\nu}\right)+\left(m_{\mu} m_{\nu}-\frac{\mu}{\mu-\nu} m_{\mu}\right)+\left(\frac{\nu}{\mu-\nu}\right)\left(\frac{\lambda}{\mu-\nu}\right)\right] \bar{H}_{\lambda} .
\end{aligned}
$$

And this requires $m_{\mu} m_{\nu}-(\mu /(\mu-\nu)) m_{\mu}+(\nu /(\mu-\nu))(\lambda /(\lambda-\nu))=0$.

But this is certainly not true for arbitrary $\lambda$. Therefore we must conclude that $d=m$.

The proof by Chover, Wainger and Ney uses Banach algebra elements and relations defined by asymptotic tails. It is similar to our proof in that it also shows certain relationships must hold in a range defined by $m$. However, it uses complex valued transforms and Cauchy's theorem. Additionally, they prove part of our Theorem 2.13 before proving $d=m$. Embrechts (1983) gives another theorem similar to Theorem 2.9, except that it refers to the probabilities $f_{n}=F\{n\}$ of a distribution with mass on the integers. His proof relies on a real analytic theorem by Rudin (1973).

(Because $\overline{F * F} \sim 2 d \bar{F}$ implies $d=m_{F}(\alpha)$ when $F \in L_{\alpha}$, one might also suspect that $\bar{F}^{* n} \sim d_{n} \bar{F}$ is equally sufficient. The answer to this ultimately depends 
on whether the bounds in Corollary 2.6(ii) are in fact sharp. For example, $\bar{F}^{* n} \sim d_{n} \bar{F}$ implies $\overline{F * F} \leq 2 d F$ for some $d$, and this alone is sufficient to prove, for Theorem 2.9,

$$
\frac{1}{2 d-m} \leqslant \lambda_{0}=\lambda_{1}=\frac{1}{m} .
$$

The remaining inequality, however, can only be resolved if one knows that

$\lim _{t \rightarrow \infty} \sup \frac{\overline{F * F}(t)}{\bar{F}(t)}=2 d \quad$ implies $\quad \lim _{t \rightarrow \infty} \sup \frac{\bar{F}^{* n}(t)}{\bar{F}(t)}=\frac{(2 d-m)^{n}-m^{n}}{2(d-m)}$.)

Knowing that $d=m$, we may revise several of our earlier results, crediting those who have previously stated them in this form.

Corollary 2.10 (Embrechts AND Goldie, 1982). If $F_{1} \in S_{\alpha}$ and $\bar{F}_{2} \sim k \bar{F}_{1}$ for some $k>0$, then $F_{2} \in S_{\alpha}$ and $F_{1} * F_{2} \in S_{\alpha}$. If $\bar{F}_{2}=o\left(\bar{F}_{1}\right)$, then $F_{1} * F_{2} \in S_{\alpha}$.

Proof. Corollaries 2.5 and 2.7 and Theorem 2.9.

Corollary 2.11 (Chover, Wainger and Ney, 1973a, b; Embrechts and GOLDIE, 1982). The following are equivalent.

(i) $F \in S_{\alpha}$.

(ii) $F \in L_{\alpha}$ and $\bar{F}^{* n} \sim n m^{n-1} \bar{F}$ for some (hence all) $n$.

(iii) $F \in L_{\alpha}$ and $F^{* n} \in S_{\alpha}$ for some (hence all) $n$.

Proof. If $F \in S_{\alpha}$, then $F \in L_{\alpha}$ and $\overline{F * F} \sim 2 m \bar{F}$ from Theorem 2.9. By Corollary $2.6, \bar{F}^{* n} \sim n m^{n-1} \bar{F}$. The remainder of the proof is virtually the same as that for Theorem 2.13 and Corollary 2.14 , which we provide later.

The remarks following Theorem 2.13 apply equally well to Corollary 2.11 , which is a special case.

Lemma 2.12 (Chover, WaINGer AND Ney, 1973b). If $F \in S_{\alpha}$, then for each $\varepsilon>0$ and some $K_{\varepsilon}, \bar{F}^{* n}(t) \leqslant K_{\varepsilon}(m+\varepsilon)^{n-1} \bar{F}(t)$ all $n$, all $t$.

Proof. Lemma 2.8 and Theorem 2.9.

The next theorem both strengthens and generalizes the known results for distributions of random sums.

TheOrem 2.13. Assume $F \in L_{\alpha}, m=\int_{0}^{\infty} e^{\alpha u} F(d u)$. Let $\left\{\lambda_{n}\right\}$ be a sequence of nonnegative coefficients such that $\lambda_{j}>0$ for some $j>1$ and $\sum_{0}^{\infty} \lambda_{n}(m+\varepsilon)^{n}<\infty$ for some $\varepsilon>0$. Denote $H=\sum_{0}^{\infty} \lambda_{n} F^{* n}$. The following are equivalent.

(i) $F \in S_{\alpha}$. 
(ii) $\bar{H} \sim c \bar{F}$ for $c=\sum_{1}^{\infty} n \lambda_{n} m^{n-1}$.

(iii) $H \in S_{\alpha}$ and $\bar{F} \neq o(\bar{H})$.

Proof. That (i) implies (ii) follows by dominated convergence using Corollary 2.11 and Lemma 2.12. By Corollary 2.5 , it immediately follows that (i) implies (iii).

To show that (ii) implies (i) we use Corollary 2.6(i). Assume $\lambda_{j}>0$ for some $j \geq 2$. Then $\bar{F}^{* j} \geq j m^{j-1} \bar{F}$ and

$$
\begin{aligned}
\lambda_{j} \bar{F}^{* j} & =\bar{H}-\sum_{n \neq j} \lambda_{n} \bar{F}^{* n} \\
& \leq c \bar{F}-\sum_{n \neq j} n \lambda_{n} m^{n-1} \bar{F} \\
& =j \lambda \lambda_{j} m^{j-1} \bar{F} .
\end{aligned}
$$

Thus $\bar{F}^{* j} \sim j m^{j-1} \bar{F}$. Also from Corollary 2.6(ii) $\bar{F}^{* j} \geq m^{j-2} \overline{F * F}+$ $(j-2) m^{j-1} \bar{F}$, so that by Lemma $2.1(\mathrm{i}, \mathrm{ii}), \overline{F * F} \leq 2 m \bar{F}+m^{2-j} \bar{F}^{* j}-j m \bar{F} \sim$ $2 m \bar{F}$. This shows $F \in S_{\alpha}$, which is (i).

To show that (iii) implies (i), we first note that according to Corollary 2.6, $\bar{F}^{* n} \geq n m^{n-1} \bar{F}$ for all $n$. Hence $H \geq\left(\sum_{0}^{\infty} n \lambda_{n} m^{n-1}\right) \bar{F}=c \bar{F}$. However, $\bar{F} \neq o(\bar{H})$, so by Corollary 3.2(i) in the next section, $F \in S_{\alpha}$.

In case $\alpha=0$, the assumption $F \in L_{0}$ may be replaced by $m=\bar{F}(0)$ (and $F \in S_{0}$ by $\overline{F * F}-2 m \bar{F}$, similarly for $H$ ). Indeed, as remarked following Lemma 2.2, $F \in L_{0}$ is a consequence of $\overline{F * F} \sim 2 m \bar{F}$ (and $H \in L_{0}$ of $\overline{H * H} \sim 2 \bar{H}(0) \bar{H}$ ). The same remark allows us to use Corollary 2.6(i) to show (ii) implies (i). As Embrechts and Goldie (1982) point out, the requirement $F \in L_{\alpha}$ when $\alpha>0$ seems to be related to the unsolved question of whether $\overline{F * F} \sim 2 d \bar{F}$ actually implies $F \in L_{\alpha}$ for some $\alpha$.

Chover, Wainger and Ney (1973) have already shown that (i) implies (ii) in Theorem 2.13, while Embrechts and Goldie (1982) and Embrechts, Goldie and Veraverbeke (1979) proved the equivalency of all three statements for the special cases where the $\lambda_{n}$ are Poisson and geometric probabilities. We now show that for these cases and for the case $H=F^{* n}$ the assumption $\bar{F} \neq o(\bar{H})$ in (iii) is unnecessary.

Corollary 2.14. Suppose $F \in L_{\alpha}, H=\sum_{0}^{\infty} \lambda_{n} F^{* n}$ and $H \in S_{\alpha}$. Then each of the following implies $F \in S_{\alpha}$.

(i) $\lim _{n \rightarrow \infty} \sup \left(\lambda_{n+1}\right) / \lambda_{n}<1 / m$.

(ii) For $q=m_{H}(\bar{F}(0) / \bar{H}(0)), \sum_{0}^{\infty} \lambda_{n}(q+\varepsilon)^{n}<\infty$ for some $\varepsilon>0$. 
Proof. For both conditions, the proof relies on Theorem 2.13 and the demonstration that $\bar{F} \neq o(\bar{H})$.

(i) Clearly, $\sum_{0}^{\infty} \lambda_{n}(m+\varepsilon)^{n}<\infty$ for some $\varepsilon>0$, as required. Assume $\bar{F}=$ $o(\bar{H})$. Then if $\bar{F} \leq \varepsilon \bar{H}$ and $\bar{F}^{* n} \leq \varepsilon k_{n} \bar{H}$, Lemma 2.4(i) yields,

$$
\begin{aligned}
\bar{F}^{* n+1} & \leq \bar{F}^{* n+1}+2 \varepsilon^{2} k_{n} m_{H} \bar{H} \\
& \leq \varepsilon^{2} k_{n} \overline{H * H}+m^{n} \bar{F}+m \bar{F}^{* n} \\
& \leq \varepsilon\left[m^{n}+m k_{n}+2 \varepsilon m_{H} k_{n}\right] \bar{H},
\end{aligned}
$$

since $\overline{H * H} \sim 2 m_{H} \bar{H}$. Hence, $\bar{F}=o(\bar{H})$ implies $\bar{F}^{* n}=o(\bar{H})$ for all $n$. Modifying a finite number of the $\lambda_{n}$, therefore, will not change the assumption $H \in S_{\alpha}$. We thus assume that $\lambda_{0}=0$ and $(m+\varepsilon) \lambda_{n+1} \leqslant \lambda_{n}$ for all $n \geqslant 1$. From Corollary 2.7, $\bar{F}=o(\bar{H})$ implies $\overline{H * F} \sim m \bar{H}$. Thus

$$
\begin{aligned}
\bar{H} & =\sum_{1}^{\infty} \lambda_{n} \bar{F}^{* n}=\lambda_{1} \bar{F}+\sum_{1}^{\infty} \lambda_{n+1} \bar{F}^{* n+1} \\
& \leqslant \lambda_{1} \bar{F}+\frac{1}{m+\varepsilon} \overline{H * F} \\
& \sim \frac{m}{m+\varepsilon} \bar{H} .
\end{aligned}
$$

This contradiction demonstrates that $\bar{F} \neq o(\bar{H})$.

(ii) We assume without any loss that $\bar{F}(0)=1, \lambda_{0}=0$ and $\Sigma_{1}^{\infty} \lambda_{n}=1$ (so that $\bar{H}(0)=1$, also). Since $\bar{F}(t) \leqslant \bar{F}^{* n}(t)$, then for all $t, \bar{F}(t) \leqslant \sum_{1}^{\infty} \lambda_{n} \bar{F}^{* n}(t)=\bar{H}(t)$ and $\bar{F}^{* n}(t) \leqslant \bar{H}^{* n}(t)$. By Lemma 2.12 , applied to $H, \bar{F}^{* n}(t) \leqslant \bar{H}^{* n}(t) \leqslant$ $K_{\varepsilon}\left(m_{H}+\varepsilon\right)^{n-1} \bar{H}(t)$. If we further assume $\bar{F}=o(\bar{H})$, then, as above, $\bar{F}^{* n}=o(\bar{H})$ and dominated convergence yields

$$
1=\lim _{t \rightarrow \infty} \frac{\bar{H}(t)}{\bar{H}(t)}=\sum_{1}^{\infty} \lambda_{n} \lim _{t \rightarrow \infty} \frac{\bar{F}^{* n}(t)}{\bar{H}(t)}=0,
$$

a contradiction. Thus $\bar{F} \neq o(\bar{H})$.

\section{Closure and factorization}

This section primarily improves results in Embrechts and Goldie (1980) for subexponential distributions and generalizes them to include exponential tail distributions. We investigate two properties for distributions $F_{j} \in S_{\alpha}$,

$$
\begin{aligned}
& \text { closure (under } * \text { ): } F_{1} * F_{2} \in S_{\alpha}, \\
& \text { factorization: } \overline{F_{1} * F_{2}} \sim m_{2} \bar{F}_{1}+m_{1} \bar{F}_{2} .
\end{aligned}
$$


We show in Theorem 3.4, as did Embrechts and Goldie for $S_{0}$, that these properties are equivalent when both $F_{1}, F_{2} \in S_{\alpha}$. Although we still cannot verify that $S_{\alpha}$ is in fact closed under convolutions, we can provide fairly general conditions on $F_{1}$ and $F_{2}$ for which the property holds.

We start with a useful lemma.

Lemma 3.1. Suppose $F_{1}, F_{2} \in L_{\alpha}, F_{3} \in S_{\alpha}$. If $\bar{F}_{1} \leqslant \bar{F}_{3}, \bar{F}_{2} \leq \bar{F}_{3}$ and $F_{3} \leqslant$ $k \bar{F}_{1} * F_{2}$ for some $k<\infty$, then each of the following holds.

(i) $F_{1} * F_{2} \in S_{\alpha}$.

(ii) $\overline{F_{1} * F_{2}} \sim m_{2} \bar{F}_{1}+m_{1} \bar{F}_{2}$.

(iii) At least one of $F_{1}, F_{2}$ is in $S_{\alpha}$. The other, if not in $S_{\alpha}$, has an asymptotically negligible tail.

Proof. We obtain (ii) using first Lemma $2.4(\mathrm{i}), \overline{F_{1} * F_{2}}+2 m_{3} \bar{F}_{3} \leq \overline{F_{3} * F_{3}}+$ $\left(m_{2} \bar{F}_{1}+m_{1} \bar{F}_{2}\right)$. Thus, by Lemma $2.1(\mathrm{i}), \overline{F_{1} * F_{2}} \leqslant\left(m_{2} \bar{F}_{1}+m_{1} \bar{F}_{2}\right)$. And by Lemma 2.2(ii), this is sufficient for $\bar{F}_{1} * F_{2} \sim m_{2} \bar{F}_{1}+m_{1} \bar{F}_{2}$. We can also show $\overline{F_{1} * F_{2}} \sim m_{2} \bar{F}_{1}+m_{1} \bar{F}_{2} \leq\left(m_{2}+m_{1}\right) \bar{F}_{3}$ and $\bar{F}_{3} \leq k_{1}\left(\bar{F}_{1} * F_{2}\right){ }^{* 2}$ for $k_{1}=$ $k / \bar{F}_{1}(0) \bar{F}_{2}(0)$. Therefore, a repeat argument gives $\left(\overline{F_{1} * F_{2}}\right)^{* 2} \sim 2 m_{1} m_{2} \overline{F_{1} * F_{2}}$. That is, $\overline{F_{1} * F_{2}} \in S_{\alpha}$.

Because of the equivalency, $m_{2} \bar{F}_{1}+m_{1} \bar{F}_{2} \in S_{\alpha}$ also. Therefore,

$$
\begin{aligned}
4 m_{1} m_{2}\left(m_{2} \bar{F}_{1}+m_{1} \bar{F}_{2}\right) & \sim m_{2}^{2} \overline{F_{1} * F_{1}}+2 m_{1} m_{2} \overline{F_{1} * F_{2}}+m_{1}^{2} \overline{F_{2} * F_{2}} \\
& \sim 2 m_{1} m_{2}\left(m_{2} \bar{F}_{1}+m_{1} \bar{F}_{2}\right)+m_{2}^{2} \overline{F_{1} * F_{1}}+m_{1}^{2} \overline{F_{2} * F_{2}} .
\end{aligned}
$$

This implies

$$
2 m_{1} m_{2}^{2} \bar{F}_{1}+2 m_{2} m_{1}^{2} \bar{F}_{2}-m_{2}^{2} \overline{F_{1} * F_{1}}+m_{1}^{2} \overline{F_{2} * F_{2}} .
$$

But each term on the left is dominated by the corresponding term on the right, asymptotically. Thus by Lemma 2.1(iv), either $2 m_{1} \bar{F}_{1} \sim \bar{F}_{1} * F_{1}$ or $2 m_{2} \bar{F}_{2}$ $\sim \overline{F_{2} * F_{2}}$, and if not both, then one set of terms must be negligible with respect to the other. In other words, we must have one of

$$
\begin{array}{ll}
F_{1} \in S_{\alpha}, & F_{2} \in S_{\alpha}, \\
F_{1} \in S_{\alpha}, & \overline{F_{2} * F_{2}}=o\left(\bar{F}_{1}\right), \quad \text { or } \\
F_{2} \in S_{\alpha}, & \overline{F_{1} * F_{1}}=o\left(\bar{F}_{2}\right) .
\end{array}
$$

This immediately gives the following corollary which provides one way to determine if a distribution is in $S_{\alpha}$.

CoRollary 3.2. Suppose $F_{1} \in S_{\alpha}$ and $F_{2} \in L_{\alpha}$.

(i) If $\bar{F}_{2} \leq \bar{F}_{1}$, then $F_{1} * F_{2} \in S_{\alpha}$. If $\bar{F}_{2} \neq o\left(\bar{F}_{1}\right)$ also, then $F_{2} \in S_{\alpha}$.

(ii) If $\bar{F}_{1} \leq \bar{F}_{2}$, then $F_{1} * F_{2} \in S_{\alpha}$ if and only if $F_{2} \in S_{\alpha}$. 
Proof. (i) Follows from Lemma 3.1 with $F_{3}=F_{1}$.

(ii) Suppose $F_{2} \in S_{\alpha}$. Then $F_{1} * F_{2} \in S_{\alpha}$ by (i).

If instead we assume $F_{1} * F_{2} \in S_{\alpha}$, then using Lemma 3.1 with $F_{3}=F_{1} * F_{2}$, we must have $F_{2} \in S_{\alpha}$ since $\bar{F}_{2} \neq o\left(\bar{F}_{1}\right)$.

We have already used Corollary 3.2 in the proof of Theorem 2.13 .

The next lemma is interesting for its simplicity.

Lemma 3.3. Suppose $F_{1}, F_{2} \in S_{\alpha}$. Let $G=F_{1} * F_{2}, H=m_{2} F_{1}+m_{1} F_{2}$. Then

$$
\begin{gathered}
\overline{H * H} \sim 2 m_{1} m_{2} \bar{G}+2 m_{1} m_{2} \bar{H}, \\
\overline{H * G} \sim 4 m_{1} m_{2} \bar{G}-m_{1} m_{2} \bar{H}
\end{gathered}
$$

and

$$
\overline{G * G} \sim 4 m_{1} m_{2} \bar{G}-2 m_{1} m_{2} \bar{H} .
$$

Proof. Straightforward application of $\overline{F_{j} * F_{j}} \sim 2 m_{j} \bar{F}_{j}$ and Lemma 2.4 .

We now provide the primary theorem for this section. Under the assumption that $F_{1}, F_{2} \in S_{0}$, Embrechts and Goldie (1980) proved a similar theorem, namely that (i), (ii) and (iv) are equivalent. In addition to proving the theorem for all classes $S_{\alpha}$, our contribution has been to weaken the assumptions and to weaken (ii) to (v).

TheOREM 3.4. Suppose $F_{1}, F_{2} \in L_{\alpha}, G=F_{1} * F_{2}, H=m_{2} F_{1}+m_{1} F_{2}$. Then the following are equivalent.

(i) $G \in S_{\alpha}$.

(ii) $\bar{G} \sim \bar{H}$, with (vi) below.

(iii) $H \in S_{\alpha}$.

(iv) $a_{1} F_{1}+a_{2} F_{2} \in S_{\alpha}$ for some (hence all) $a_{1}, a_{2}>0$.

(v) $\bar{G} \sim a_{1} \bar{F}_{1}+a_{2} \bar{F}_{2}$ for some $a_{1}, a_{2}$, with (vi) below.

Statements (ii) and (v) refer to

(vi) At least one of $F_{1}$ and $F_{2}$ is in $S_{\alpha}$. The other, if not in $S_{\alpha}$, has an asymptotically negligible tail.

Proof. Note that (ii) implies (v) because (ii) is a special case of (v).

That (i) implies (ii) (and (v)) follows immediately from Lemma 3.1.

On the other hand (ii) implies (i), because if $F_{1} \in S_{\alpha}$ and $\bar{F}_{2}=o\left(\bar{F}_{1}\right)$, then $F_{1} * F_{2} \in S_{\alpha}$ by Corollary 2.10. And if $F_{1}, F_{2} \in S_{\alpha}$ then by Lemma 3.3, $\overline{G * G} \sim$ $4 m_{1} m_{2} \bar{G}-2 m_{1} m_{2} \bar{H} \sim 2 m_{1} m_{2} \bar{G}$. 
Furthermore, (i) implies (iv) (with "all") when $F_{1}, F_{2} \in S_{\alpha}$, since

$$
\begin{aligned}
\left(a_{1} \bar{F}_{1}+a_{2} \bar{F}_{2}\right)^{* 2} & =a_{1}^{2} \overline{F_{1} * \bar{F}_{1}}+2 a_{1} a_{2} \bar{G}+a_{2}^{2} \overline{F_{2} * F_{2}} \\
& \sim 2\left(a_{1} m_{1}+a_{2} m_{2}\right)\left(a_{1} \bar{F}_{1}+a_{2} \bar{F}_{2}\right) .
\end{aligned}
$$

When $F_{1} \in S_{\alpha}, \bar{F}_{2}=o\left(\bar{F}_{1}\right)$, (iv) holds because $a_{1} \bar{F}_{1}+a_{2} \bar{F}_{2} \sim a_{1} \bar{F}_{1}$. To show that (iv) (with "some") implies (i), let $c=\max \left(a_{1} / m_{2}, a_{2} / m_{1}\right)$ and $K=a_{1} F_{1}+a_{2} F_{2}$. Then $a_{1} \bar{F}_{1} \leq \bar{K}, a_{2} \bar{F}_{2} \leq \bar{K}$ and $\bar{K} \leqslant c \bar{H} \leqslant c \bar{G}$. Applying Lemma 3.1, it follows that $K \in S_{\alpha}$ implies $G \in S_{\alpha}$. Statement (iii) is equivalent to (i) because (iv) is.

It remains only to show that (v) implies (i). Assume without loss that $F_{1} \in S_{\alpha}$. Note first that if $\bar{F}_{2} \sim k \bar{F}_{1}$, then $G \in S_{\alpha}$ by Corollary 2.10 . So we assume $\bar{F}_{2}+k \bar{F}_{1}$ for any $k$ (finite, zero or infinite) and we must also assume $F_{2} \in S_{\alpha}$, since (vi) holds. Note that $m_{2} \bar{F}_{1}+m_{1} \bar{F}_{2} \leqslant a_{1} \bar{F}_{1}+a_{2} \bar{F}_{2}$. Since (ii) implies (i), the result is obvious if $a_{1}=m_{2}, a_{2}=m_{1}$. If $a_{2}<m_{1}$, then by Lemma 2.1(i), $m_{2} \bar{F}_{1}+\left(m_{1}-a_{2}\right) \bar{F}_{2} \leqslant a_{1} \bar{F}_{1}$, which requires $a_{1} \geqslant m_{2}$. Thus

$$
\bar{F}_{2} \leq\left(a_{1}-m_{2}\right) /\left(m_{1}-a_{2}\right) \bar{F}_{1} \text {. }
$$

By Corollary 3.2(i), $G \in S_{\alpha}$. (Note that in fact $a_{1} \bar{F}_{1}+a_{2} \bar{F}_{2} \sim \bar{G} \sim m_{2} \bar{F}_{1}+m_{1} \bar{F}_{2}$. This implies $a_{1}=m_{2}, a_{2}=m_{1}$ since $\bar{F}_{1}$ and $\bar{F}_{2}$ are not asymptotically equivalent (Lemma 2.1(iii)).) The case $a_{1}<m_{2}$ is handled similarly. On the other hand, we intend to show that $a_{1} a_{2}=m_{1} m_{2}$ and thus no other cases are possible.

Since $\bar{G} \sim a_{1} \bar{F}_{1}+a_{2} \bar{F}_{2}$ and $\bar{F}_{j} * F_{j} \sim 2 m_{j} \bar{F}_{j}$, then from Lemma 2.4(ii),

$$
\begin{aligned}
\overline{G * G} & \sim a_{1}^{2} \overline{F_{1} * F_{1}}+2 a_{1} a_{2} \bar{G}+a_{2}^{2} \overline{F_{2} * F_{2}}+2\left(m_{1} m_{2}-a_{1} m_{1}-a_{2} m_{2}\right) \bar{G} \\
& \sim 2 a_{1}\left(a_{1} a_{2}+m_{1} m_{2}-a_{2} m_{2}\right) \bar{F}_{1}+2 a_{2}\left(a_{1} a_{2}+m_{1} m_{2}-a_{1} m_{1}\right) \bar{F}_{2} .
\end{aligned}
$$

By Lemma 3.3, another asymptotic expression for $\overline{G * G}$ is

$$
\begin{aligned}
\overline{G * G} & \sim 4 m_{1} m_{2} \bar{G}-2 m_{1} m_{2} \bar{H} \\
& \sim 2 m_{1} m_{2}\left(2 a_{1}-m_{2}\right) \bar{F}_{1}+2 m_{1} m_{2}\left(2 a_{2}-m_{1}\right) \bar{F}_{2} .
\end{aligned}
$$

Since $\bar{F}_{1}$ and $\bar{F}_{2}$ are not asymptotically equivalent, by Lemma 2.1(iii) the respective coefficients in (3.1) and (3.2) equal. That is,

$$
a_{1}\left(a_{1} a_{2}+m_{1} m_{2}-a_{2} m_{2}\right)=m_{1} m_{2}\left(2 a_{1}-m_{2}\right)
$$

and

$$
a_{2}\left(a_{1} a_{2}+m_{1} m_{2}-a_{1} m_{1}\right)=m_{1} m_{2}\left(2 a_{2}-m_{1}\right) .
$$

These reduce to $\left(a_{1}-m_{2}\right)\left(a_{1} a_{2}-m_{1} m_{2}\right)=0$ and $\left(a_{2}-m_{1}\right)\left(a_{1} a_{2}-m_{1} m_{2}\right)=$ 0 , requiring at least $a_{1} a_{2}=m_{1} m_{2}$ and justifying our argument above.

For $F_{1}, F_{2} \in L_{\alpha}$, Theorem 3.4 characterizes the case $G \in S_{\alpha}$ and the case $a_{1} F_{1}+a_{2} F_{2} \in S_{\alpha}$. It does not however characterize the case $\bar{G} \sim \bar{H}$ without additional assumptions. This suggests there may be examples for which $F_{1} * F_{2}$ is not in $S_{\alpha}$, yet we still have $\overline{F_{1} * F_{2}} \sim m_{2} \bar{F}_{1}+m_{1} \bar{F}_{2}$ and $F_{1}, F_{2} \in L_{\alpha}$. 
In Corollary 3.2 we have given a condition for $F_{1} * F_{2}$ to be in $S_{\alpha}$ which is easily checked. We will now give a more general condition.

Theorem 3.5. Suppose $F_{1}, F_{2} \in S_{\alpha}$. Define $b(t)=\bar{F}_{1}(t) / \bar{F}_{2}(t)$. If for some $x_{1}>1>x_{0}>0, \sup _{t} \sup _{x_{0}<x<x_{1}} b(x t) / b(t)<\infty$, then $F_{1} * F_{2} \in S_{\alpha^{*}}$ In particular, if $b(t) \in R V_{\rho}$ for any $\rho$, then $F_{1} * F_{2} \in S_{\alpha}$.

PROof. The condition on $b$ is called the R-O variation property (Seneta (1976), Appendix). Since $x_{0}<1<x_{1}$, the values of $x_{0}$ and $x_{1}$ may be extended arbitrarily. We set $K=\sup _{t} \sup _{1 / 2 \leqslant x \leqslant 2} b(x t) / b(t)$.

Thus, for $0 \leqslant u \leqslant t / 2$,

$$
\frac{\bar{F}_{1}(t-u)}{\bar{F}_{1}(t)} \leqslant K \frac{\bar{F}_{2}(t-u)}{\bar{F}_{2}(t)} .
$$

Since $F_{2} \in S_{\alpha}$, then $\overline{F_{2} * F_{2}} \sim 2 m_{2} \bar{F}_{2}$ so that

$$
\lim _{t \rightarrow \infty} \int_{0}^{t / 2} \frac{\bar{F}_{2}(t-u)}{\bar{F}_{2}(t)} F_{2}(d u)=m_{2}=\int_{0}^{\infty} \lim _{t \rightarrow \infty}\left[\frac{\bar{F}_{2}(t-u)}{\bar{F}_{2}(t)} 1_{[0, t / 2]}(u)\right] F_{2}(d u)
$$

and $\lim _{t \rightarrow \infty} \bar{F}_{2}^{2}(t / 2) / \bar{F}_{2}(t)=0$. Therefore, by dominated convergence

$$
\lim _{t \rightarrow \infty} \int_{0}^{t / 2} \frac{\bar{F}_{1}(t-u)}{\bar{F}_{1}(t)} F_{2}(d u)=m_{2} .
$$

We also have

$$
\lim _{t \rightarrow \infty} \frac{\bar{F}_{1}(t / 2) \bar{F}_{2}(t / 2)}{\bar{F}_{1}(t)} \leqslant K \lim _{t \rightarrow \infty} \frac{\bar{F}_{2}^{2}(t / 2)}{\bar{F}_{2}(t)}=0 .
$$

And by a similar argument

$$
\lim _{t \rightarrow \infty} \int_{0}^{t / 2} \frac{\bar{F}_{2}(t-u)}{\bar{F}_{2}(t)} F_{1}(d u)=m_{1} .
$$

Thus $\overline{F_{1} * F_{2}}(t) \sim m_{2} \bar{F}_{1}(t)+m_{1} \bar{F}_{2}(t)$ and $F_{1} * F_{2} \in S_{\alpha}$.

With similar conditions, one may easily discuss tails of finitely many convolutions. Results for infinite convolutions are forthcoming in another paper.

\section{Conclusion}

Except for Theorem 3.5, all of our results relied on the Lemmas 2.1-2.4 and 2.8. These were the only results which required analytic proofs. This has made it possible to generalize results and, in particular to obtain a real analytic proof of the basic result, Theorem 2.9. Our method, however, relies heavily on the 
monotonicity of the distribution tails. Embrechts (1983) used the work of Rudin (1973) to get a real analytic proof when $f$ is the atomic density of a lattice distribution $F$. Letting $f_{n}=F\{n\}$ and $f_{n} * f_{n}=F^{*} F\{n\}$, then Embrechts' result is

$$
\text { If } f_{n} \sim R f_{n+1} \text { and } f_{n} * f_{n} \sim 2 d f_{n}, \text { then } d=\sum_{0}^{\infty} R^{n} f_{n} .
$$

However, it is also apparent that $f_{n}=\bar{F}(n)-\bar{F}(n+1) \sim(1-R) \bar{F}(n)$. Thus, for $R<1$, Embrechts' result is a special case of ours.

Chover, Wainger and Ney (1973a) proved the lattice result using a Banach algebra technique. Their technique also gave a result for the density $f$ of absolutely continuous $F$, namely:

$$
\text { If } f(t) \sim e^{\alpha u} f(t+u) \text { and } f * f(t) \sim 2 d f(t) \text {, then } d=\int_{0}^{\infty} e^{\alpha u} f(u) d u \text {. }
$$

This is different from Theorem 2.9 for the density case, because $F \in L_{\alpha}$ is necessary but not sufficient for $f(t) \sim e^{a u} f(t+u)$. We have not yet obtained a real analytic proof for the density version.

\section{Acknowledgement}

The referee's careful critique is greatly appreciated.

\section{References}

V. P. Chistyakov (1964), 'A theorem on sums of independent positive random variables and its applications to branching random processes,' Theory Probab. Appl. 9, 640-648.

J. Chover, P. Ney and S. Wainger (1973a), 'Functions of probability measures,' J. Analyse Math. 26, 255-302.

J. Chover, P. Ney and S. Wainger (1973b), 'Degeneracy properties of subcritical branching process,' Ann Probab. 1, 663-673.

D. B. H. Cline (1986), 'Convolution tails, product tails and domains of attraction', Probab. Theor. Rel. Fields 72, 525-557.

R. A. Davis and S. I. Resnick (1985a), 'Limit theory for moving averages of random variables with regularly varying tail probabilities,' Ann. Probab. 13, 179-195.

R. A. Davis and S. I. Resnick (1985b), 'More limit theory for the sample correlation function of moving averages', Stochastic Process. Appl. 20, 257-279.

P. Embrechts (1983), 'The asympotic behaviour of series and power series with positive coefficients', Verh. Konink. Acad. Wetensch., Belgie.

P. Embrechts and C. M. Goldie (1980), 'On closure and factorization properties of subexponential distributions', J. Aust. Math. Soc. (Ser. A) 29, 243-256. 
P. Embrechts and C. M. Goldie (1982), 'On convolution tails,' Stochastic Process. Appl. 13, 263-278.

P. Embrechts, C. M. Goldie and N. Veraverbeke (1979), 'Subexponentially and infinite divisibility', $Z$. Wahrsch. Verw. Gebiete 49, 335-347.

W. Feller (1971), An introduction to probability theory and its applications, Vol. II (Wiley).

W. Rudin (1973), 'Limits of ratios of tails of measures', Ann. Probab. 1, 982-994.

E. Seneta (1976), Regularly varying functions (Lecture Notes in Mathematics 508, Springer-Verlag).

\section{Department of Statistics}

Texas A \& M University

College Station, Texas 77843

U.S.A. 